\section{Empfehlungen}

für die Diagnostik

und Therapie

psychotischer
Dies ist eine interdisziplinäre und interprofessionelle Guideline, die durch Vertreter mehrerer Institutionen ent-

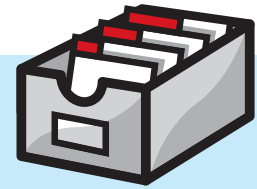
wickelt wurde. Sie wurde für «Praxis» zusätzlich einem externen Reviewing unterzogen.

Sollten Sie anderer Meinung sein als hier berichtet, schreiben Sie uns unter redaktion.praxis@hogrefe.ch.

\title{
Erkrankungen im Alter
}

\section{Recommendations for the Diagnosis and Therapy of Psychotic Disorders in the Elderly}

Stefan Klöppel ${ }^{1,13}$, Egemen Savaskan²,13, Jean-Marie Annoni3,14, Jean-Luc Berruex³, Livia Bohli ${ }^{4}$, Maria Eder ${ }^{1}$, Ulrich Hemmeter ${ }^{5,13}$, Therese Hirsbrunner ${ }^{6,15}$, Thomas Leyhe ${ }^{12,7, \dagger}$, Annetta Neyenhuys ${ }^{12}$, Julius Popp 2,8, Katrin Rauen², Jean-Pierre Schuster ${ }^{8}$, Vita Sulaj9,13,17, Samuel Vögeli1 ${ }^{2,15}$, Armin von Gunten ${ }^{8,13}$, Maxim Zavorotnyy ${ }^{10,11,17}$, Leonardo Zullo ${ }^{8}$, Fabienne Roth ${ }^{12,15,16}$ und Claudia Lötscher ${ }^{12,15,16}$

\footnotetext{
Universitätsklinik für Alterspsychiatrie und Psychotherapie, Universität Bern, Bern

2 Klinik für Alterspsychiatrie, Psychiatrische Universitätsklinik Zürich, Zürich

${ }^{3}$ Département des Neurosciences, Université et Hôpital de Fribourg, Fribourg

${ }^{4}$ Privatklinik, Aadorf

5 Psychiatrie St. Gallen Nord, St. Gallen

${ }^{6}$ Solothurner Spitäler AG, Psychiatrische Dienste, Solothurn

7 Universitäre Altersmedizin Felix Platter, Basel

${ }^{3}$ Service Universitaire de Psychiatrie de l'Âge Avancé, CHUV, Prilly-Lausanne

${ }^{9}$ Psychiatrische Dienste Aargau AG, Klinik für Konsiliar, Alters- und Neuropsychiatrie, Windisch

${ }^{10}$ Psychiatrische Dienste Aargau AG, Klinik für Psychiatrie und Psychotherapie, Windisch

"Klinik für Psychiatrie und Psychotherapie der Philipps-Universität Marburg, Marburg, Deutschland

${ }^{12}$ Universitäre Psychiatrische Kliniken, Basel

${ }^{3}$ Schweizerische Gesellschaft für Alterspsychiatrie (SGAP), Weggis

${ }^{14}$ Schweizerische Neurologische Gesellschaft (SNG), Basel

${ }^{15}$ Schweizerischer Verein für Pflegewissenschaft (VfP): Akademische Fachgesellschaft für gerontologische Pflege und Akademische Fachgesellschaft für psychiatrische Pflege, Bern

${ }^{16}$ Schweizer Berufsverband der Pflegefachfrauen und Pflegefachmännern (SBK Schweiz/ASI Suisse), Bern

${ }^{17}$ Schweizerische Gesellschaft für Interventionelle Psychiatrie (SGIP), Bern

+ Verstorben
}

\begin{abstract}
Zusammenfassung: Psychotische Erkrankungen im Alter umfassen ein grosses Spektrum an Ursachen und Manifestationen. Häufig treten sie im Rahmen einer Depression, einer demenziellen Entwicklung, eines Substanz- und Medikamentenmissbrauchs oder eines Delirs auf. Während im Alter neue Erkrankungen auftreten können, erreichen zunehmend auch mehr Personen mit langjährig bestehenden, chronisch psychotischen Störungen das höhere Lebensalter. Neben psychotischen Störungen sind im Alter kognitive und somatische Beeinträchtigungen häufig, weshalb eine Fremdanamnese unverzichtbar ist. Die krankheitsbedingt veränderten Lebensumstände, die Komplexität und die jeweils individuelle Situation gilt es bei der Diagnostik und Therapie zu integrieren. Die vorliegenden Empfehlungen sind unter der Federführung der Schweizerischen Gesellschaft für Alterspsychiatrie und -psychotherapie (SGAP) in Zusammenarbeit mit den akademischen Fachgesellschaften für gerontologische und psychiatrische Pflege des Vereins für Pflegewissenschaft und weiteren Fachverbänden entstanden. Ziel ist es, den aktuellen Stand des Wissens über die psychotischen Erkrankungen im Alter sowie über die Möglichkeiten der Diagnostik und Therapie zusammenzufassen und den interprofessionellen, klinischen Teams in der stationären und ambulanten Versorgung zur Verfügung zu stellen.
\end{abstract}

Schlüsselwörter: Psychotische Störungen, Alter, Schizophrenie, Antipsychotika, Psychosoziale Intervention, Interprofessionalität 


\begin{abstract}
Psychotic disorders in the elderly cover a wide range of causes and manifestations. They often occur as part of a depression, dementia, substance abuse or delirium. While psychosis can occur with a first manifestation in advanced age, many patients with chronic psychotic disorders reach a high age. Many elderly individuals are also affected by cognitive impairment and somatic conditions, making a third-party history most relevant. The associated changes in life and the complexity of the individual situation needs to be integrated into the diagnosis and treatment. The presented recommendations have been developed under the lead of the Swiss Society of Old Age Psychiatry (SGAP) in collaboration with the Swiss Association of Nurses (SBK) and the subcommittees for gerontological and psychiatric nursing of the association of nursing science (VFP) as well as further professional societies. We aim to make current knowledge concerning diagnosis and treatment available to the interprofessional teams working in in- and outpatients' settings.
\end{abstract}

Keywords: Psychosis, elderly, antipsychotics, schizophrenia, psychosocial intervention, interprofessionality

\begin{abstract}
Résumé: Les troubles psychotiques chez les personnes âgées ont des causes et des manifestations très diverses. Ils surviennent souvent dans le cadre d'une dépression, d'une démence, des troubles liés à l'utilisation de substances psychoactives ou d'un état confusionnel aigu. Alors qu'une psychose peut se manifester pour la première fois chez la personne âgée, un grand nombre de patients souffrant de longue date de troubles psychotiques chroniques atteignent aujourd'hui un âge avancé. Des troubles cognitifs et des affections somatiques accompagnent souvent les troubles psychotiques. Leur complexité, leurs conséquences sur le quotidien et la situation individuelle de chaque patient doivent être prises en compte dans tout effort de diagnostic et de traitement. Les recommandations présentées ici ont été élaborées sous la direction de La Société Suisse de Psychiatrie et Psychothérapie de la Personne Agée (SPPA) en collaboration avec l'Association suisse des infirmières et infirmiers (ASI) et les sous-comités des soins infirmiers gérontologiques et psychiatriques de l'Association suisse pour les sciences infirmières (APSI) ainsi que d'autres organisations professionnelles. Notre objectif est de mettre à disposition des équipes interprofessionnelles travaillant en milieu hospitalier et ambulatoire les connaissances actuelles en matière de diagnostic et de traitement des troubles psychotiques à l'âge avancé.
\end{abstract}

Mots clés: Psychose, personnes âgées, antipsychotiques, schizophrénie, intervention psychosociale, interprofessionnalité

Psychotische Erkrankungen im Alter umfassen ein grosses Spektrum hinsichtlich der Ursachen, der klinischen Manifestation, des Schweregrads und der damit verbundenen Krankheitsrelevanz sowie den Funktionseinschränkungen auf physischer, psychischer und sozialer Ebene. Das Behandlungsteam hat es also mit einer heterogenen Personengruppe zu tun, weshalb die individuelle Diagnostik und Therapie bedeutsam ist. Dies erfordert die Erarbeitung individuell abgestimmter und multiprofessionell getragener Lösungen, welche die jeweilige Lebenswelt und das Behandlungssetting der betroffenen Personen berücksichtigen.

Psychosen sind Erkrankungen mit phasenweisem Verlust des Bezugs zur Realität, die mit ausgeprägten Störungen des Denkens (z.B. Wahn), der Perzeption (z.B. Halluzinationen) und der Emotionen (z.B. Affektverflachung) einhergehen. Weiter kann die Erkrankung in Positivsymptome (Wahn, Halluzinationen, Denkstörungen, Gefühl, kontrolliert zu werden) sowie Negativsymptomen (z.B. Affektverflachung) unterteilt werden. Für ältere psychotisch erkrankte Menschen gibt es wenige systematische Behandlungsstudien. In den vorliegenden Empfehlungen wurden, so weit verfügbar, die primär bei älteren Kranken durchgeführten Studien inkludiert. Gerade bei den neueren, nicht medikamentösen und psychosozialen Therapieformen waren die Übertragung von Studienergebnissen von jüngeren Altersgruppen und der Expertenkonsens erforderlich. Als weitere Herausforderung zeigt sich die grosse Anzahl an verschiedenen Betreuungssettings (z.B. psychiatrische Kliniken, somatische Spitäler, krankenhausexterne Betreuung, Langzeiteinrichtungen etc.). Bei älteren Menschen ist es erforderlich, die jeweiligen Behandlungsempfehlungen wie Psychotherapie, die Auswahl einer medikamentösen Behandlung sowie die interventionellen Verfahren z.B. Elektrokonvulsionstherapie (EKT) dem jeweiligen Funktionsniveau und der Belastbarkeit der Betroffenen anzupassen.

Das Ziel der vorliegenden Empfehlungen ist es, die Diagnostik bei älteren Menschen mit einer Psychose zu stärken und angemessene Therapie- und Behandlungsoptionen zu skizzieren. Dabei wird den psychotherapeutischen und pflegerisch-psychosozialen Interventionsmöglichkeiten ein besonderer Stellenwert beigemessen. Diese Empfehlungen stehen als umfassendes Manual in Buchform zur Verfügung [1]. Die vorliegende Version ist eine Zusammenfassung. Die Empfehlungen sind als Erweiterung zu existierenden S3Leitlinien und Empfehlungen [2, 3, 4, 5, 6] anzusehen.

\section{Epidemiologie}

Psychotische Symptome treten im Alter bei ca. 10-26\% der Bewohnenden von Alters- und Pflegeheimen [7] auf. Am häufigsten betreffen sie Personen mit Demenz (40\%) oder depressiven Störungen (33\%). Letztere sind im Alter deutlich häufiger mit psychotischen Symptomen verbunden als bei jüngeren Erwachsenen [8]. Hier sind vor allem wahn-

Im Artikel verwendete Abkürzungen:

EKT Elektrokonvulsionstherapie

NOSGER Nurses Observation Scale für Geriatric Patients 
hafte Verkennung, Verarmungswahn, Beeinträchtigungserleben und Misstrauen als wesentliche Symptome bei schweren depressiven Störungen mit psychotischen Symptomen im Alter zu nennen. Weitere wichtige Ursachen sind Substanz- und Medikamentenmissbrauch (11\%) sowie Delirien (10\%). Deutlich seltener sind Schizophrenien Ursache einer psychotischen Störung im Alter (1\%) [7]. Neben schweren Depressionen sind Manien und schizoaffektive Störungen weitere Ursachen primärer Psychosen. Wahnvorstellungen können aber auch bei Persönlichkeitsstörungen das führende Symptom ausmachen. Insgesamt sind bei älteren Menschen sekundäre Psychosen (40\%) häufiger als primäre, weshalb eine umfassende somatische Diagnostik inklusive zerebrale Bildgebung erforderlich ist.

Die Gesamtzahl möglicher Ursachen sekundärer Psychosen ist sehr gross und stellt in der klinischen Praxis eine Herausforderung dar. Entsprechend Tabelle 1 ist es deshalb sinnvoll, die Ursachen nach ihrer Fokalität zu unterscheiden.

\section{Diagnostik psychotischer Störungen}

Das klinische Bild der Psychosen im Alter ist heterogen bezüglich der psychischen und somatischen Situation und der möglichen psychosozialen Beeinträchtigungen im Alltag. Eine differenzierte Diagnostik inklusive des zeitlichen Verlaufs und die Berücksichtigung fremdanamnestischer Angaben sind wesentlich.

Die in der Altersmedizin etablierten diagnostischen Elemente sollten zum Einsatz kommen und dem Bedarf entsprechend erweitert werden. Häufig sinnvoll sind Laborkontrolle, Bildgebung, Liquordiagnostik und der Einsatz spezifischer Assessments zur Erfassung der medizinischen und psychosozialen Funktionseinschränkungen (Tabelle 2). Grundsätzlich soll die Diagnostik störungsspezifisch durchgeführt werden. Bei differenzialdiagnostischen Überlegun-
Tabelle 1. Ursachen sekundärer Psychosen

\begin{tabular}{ll}
\hline Systemisch & $\begin{array}{l}\text { Elektrolytstörung, metabolische oder endokrine } \\
\text { Störungen, Infektionen, Intoxikationen, etc. }\end{array}$ \\
Fokale oder diffuse & $\begin{array}{l}\text { Schlaganfall, neurodegenerative Erkrankung, } \\
\text { Hirnschädigung }\end{array}$ \\
Entzündung, etc. \\
Sensorische Störung & $\begin{array}{l}\text { Visusminderung, Charles-Bonnet-Syndrom } \\
\text { (optische Halluzinationen, die meist von den } \\
\text { Betroffenen als nicht real wahrgenommen } \\
\text { werden), Hörminderung, etc. }\end{array}$ \\
\hline
\end{tabular}

gen zu Depression, Demenz und Delir müssen entsprechende Empfehlungen für die Diagnostik berücksichtigt werden $[4,6,9,10]$.

\section{Erfassung der psychosozialen Situation}

Ältere Menschen mit einer psychotischen Erkrankung, welche über einen längeren Zeitraum oder über Jahre Krankheitssymptome aufweisen, erleben oft erhebliche Beeinträchtigungen in ihren Aktivitäten des täglichen Lebens und in ihren sozialen Funktionsniveaus [11]. Die Anwendung spezifischer Assessments hilft, sowohl die spezifischen Störungen/Beeinträchtigungen/Auswirkungen akut wie chronisch psychotisch erkrankter Menschen als auch die Aktivitäts- und Funktionseinbussen im Alltag zu bestimmen. Assessments verbessern die objektive Beurteilung einer Situation und ermöglichen eine zielorientierte, multiprofessionelle Abstimmung und Behandlung. Zur Erfassung der pflegerischen psychiatrischen Phänomene wird das «Ergebnisorientierte Pflege-Assessment für das psychiatrische Setting (epaPsyC)» empfohlen. Zur Erfassung der Funktionseinbussen bei den Aktivitäten des täglichen Lebens kann unter anderem auf den «Nurses Observation Scale für Geriatric Patients», kurz NOSGER, zurückgegriffen werden. Bei Verhaltensauffälligkeiten sollte die «Verstehende Diagnostik/ABC-Verhaltensanalyse» eingesetzt werden [12].

Tabelle 2. Übersicht der diagnostischen Schritte und Assessments

\begin{tabular}{ll}
\hline Diagnostik & Kommentar \\
\hline Anamnese & Eigen- und Fremdanamnese, Vor- und Familiengeschichte, komorbide Erkrankungen, Medikamenten- und \\
& Suchtanamnese, psychosoziale Anamnese (soziale Netzwerke, finanzielle Probleme, Verlusterlebnisse, körper- \\
& liche Einschränkungen etc.) \\
& Erfassung der Risikofaktoren: genetische Prädisposition, sensorische Beeinträchtigungen, zerebrale Atrophie, \\
& Hirnverletzungen, somatische/neurologische Störungen, reduzierter Allgemeinzustand, prämorbide Persönlich- \\
& keitsstörungen, weibliches Geschlecht, soziale Faktoren wie Einsamkeit und Isolation
\end{tabular}

Untersuchung (inkl. neurologische/geriatrische Untersuchung)

Labor

Differenzialdiagnostik

Bildgebung
Differenzialblutbild, Vitamin B12, Folsäure, Schilddrüsenfunktion, differenziertes Blutbild

Bei differenzialdiagnostischen Überlegungen fakultativ: Spiegelbestimmung der eingenommenen Medikamente, arterielle Blutgasanalyse, Oxymetrie, Urinstatus, EKG, Thorax-Röntgen, weiterführende Neuropsychologie bei kognitiven Störungen 
In der stationären und ambulanten Langzeitpflege ist es für die Leistungsbewilligung und Festlegung von Entgelt verpflichtend, ein standardisiertes Instrument zur Erhebung des Pflegebedarfs anzuwenden. In der Deutschschweiz hat sich das Resident Assessment Instrument (RAI: www.qsys.ch) oder Bewohnende-Einstufungs- und Abrechnungssystems (BESA: www.besacare. ch), in der Westschweiz das Plaisir/Plex (PLAnification Informatisée des Soins Infirmiers Requis: http://www.ctplaisir.ch/) etabliert. Es empfiehlt sich, diese standardisiert angewendeten Instrumente im Langzeitbereich mit einem psychiatrisch-fokussierten Assessment zu ergänzen [13]. Tabelle 2 fasst die diagnostischen Massnahmen zusammen.

Bei der Anamnese und Diagnostik gilt es, die standardisierten Verfahren mit den Präferenzen der Betroffenen sowie die des Unterstützungssystems zu ergänzen.

\section{Folgeerkrankungen und Komorbiditäten}

Psychosen sind mit einer verkürzten Lebenserwartung verbunden. Dies gilt sowohl für Menschen, die mit einer früh begonnenen Schizophrenie alt werden, wie auch für solche, die in späteren Lebensphasen eine Psychose entwickeln. Der Zusammenhang erklärt sich durch den krankheitsbedingt ungünstigeren Lebensstil (einschliesslich des Konsums gesundheitsschädlicher Substanzen), eine erhöhte Suizidgefahr, niedrige Behandlungsraten, eine unzureichende Inanspruchnahme medizinischer Versorgungsleistungen und die negativen Folgen der medikamentösen Therapie [2]. Darüber hinaus verkürzen viele somatische Ursachen sekundärer Psychosen (z.B. neurodegenerative Erkrankungen) selber die Lebenserwartung $[11,14,15]$. Da im Alter gehäuft somatische Beschwerden auftreten, ist es Bestandteil jeder Therapie bei älteren Menschen, die Komorbiditäten zu erfassen und ihren spezifischen Effekt auf das Funktionsniveau zu bewerten [11, 14]. Dieses Erfassen ist bei Älteren aufgrund kognitiver und kommunikativer Beeinträchtigungen zusätzlich erschwert, z.B. weil die Abgrenzung zwischen psychiatrischen und somatischen Symptomen nicht deutlich wird [16]. Dies hat zur Folge, dass Chancen zur Früherkennung somatischer Komplikationen oft verpasst oder wenig genutzt werden. Eine kombinierte somatisch-psychiatrische Versorgung ist deshalb erforderlich [14].

Bei älteren Menschen mit einer psychotischen Erkrankung ist zu beachten, dass die Notwendigkeit einer Behandlung durch die Betroffenen selbst nicht immer wahrgenommen wird. Dies trifft sowohl auf Personen zu, die an einer Psychose oder psychotischen Störung leiden, wie auch auf die Begleit- bzw. Folgeerkrankungen. Die betroffene Person nimmt die notwendigen Therapien möglicherweise als bedrohlich oder gar schädlich wahr, sodass sie nicht oder nur teilweise in der Lage ist, sich z.B. mit der Pflege des Körpers, angemessener Ernährung etc. zu beschäftigen und diese im Alltag umzusetzen. Motivation zur Veränderung des Gesundheitsverhaltens gilt es in der Behandlung pro-aktiv zu unterstützen. Möglicherweise muss die Anleitung und Unterstützung im Umgang mit dem Management von somatischen Erkrankungen von Angehörigen oder Fachpersonen gewährleistet werden $[13,16]$.

Da Pflegepersonen verglichen mit anderen Gesundheitsfachpersonen in allen Versorgungssettings in einem besonders nahen und zeitlich oft langdauernden Kontakt mit psychisch erkrankten Menschen stehen, leisten sie oft einen wesentlichen Beitrag bei der Sicherstellung und Begleitung der somatischen Gesundheit der Betroffenen [13].

\section{Behandlung und Therapien}

Im Grundsatz unterscheidet sich die Festlegung von Therapiezielen bei Älteren nicht von denen bei Jüngeren. Ältere Menschen sind jedoch häufiger von einer chronischen, psychischen Erkrankung betroffen. Aufgrund der Chronizität geht es primär um die Sicherstellung und das Beibehalten eines guten Funktionsniveaus, um die Symptomreduktion, um Wohlbefinden und den Erhalt der Lebensqualität und weniger um eine vollständige Remission der Krankheitssymptome (Tabelle 3 ).

\section{Psychotherapie}

Mittlerweile steht eine Vielzahl psychotherapeutischer Ansätze zur Verfügung. Mit wenigen Ausnahmen wurden die wissenschaftlichen Studien zur Überprüfung der Wirksamkeit von Psychotherapie in Kombination mit einer antipsychotischen Pharmakotherapie durchgeführt und an Studienpopulationen des frühen oder mittleren Erwachsenenalters erforscht. In den wenigen Studien bei älteren Menschen konnte die Wirksamkeit von kognitiv-verhaltenstherapeutischen Verfahren nachgewiesen werden [17]. Eine Adaption der psychotherapeutischen Interventionen ist bei älteren Personen von Vorteil und erhöht die Wirksamkeit [18]. Folgende Parameter sollten in der Therapie älterer Menschen angepasst werden:

- Vermehrte Repetition kognitiver Therapieelemente (Psychoedukation)

- Kürzere Sitzungen

- Vermehrtes Üben

- Präsentation von Informationen in verschiedenen Sinnesmodalitäten, auch, um kognitive Einschränkungen zu kompensieren

- Integration alltagsrelevanter Probleme

Erfreulicherweise gibt es auch Faktoren, die den Erfolg einer Psychotherapie im Alter positiv beeinflussen, sogenannte «Gewinne des Alterns» [19]. Hierzu zählen:

- Die Zunahme von Reife als kumulierte Bewältigungsund Lebenserfahrung 
Tabelle 3. Mögliche Therapieziele bei älteren Menschen mit einer Psychose

\section{Übersicht}

- Verbesserung oder Erhalt des sozialen Funktionsniveaus und der Alltagskompetenzen

- Erhalt von Wohlbefinden und subjektiver Lebensqualität

- Symptomkontrolle und Symptomreduktion

- Rückfallprophylaxe

- Suizidprophylaxe und Suizidalität mindern

- Unterstützung im Alltag bei bestmöglicher Autonomie/Selbstbestimmung

- Verbesserung und Gewährleistung der Gesundheitsfürsorge und Steigerung der Motivation zur Therapieadhärenz

- Kontrolle über das eigene Leben behalten und ein hoffnungsvolles, konstruktives und befriedigendes Leben leben

- Verminderung und Umgang von Schamgefühlen und Angst vor Fremd- oder Eigenstigmatisierung

- Kontrolle bzw. Monitoring und Verminderung der komorbiden Symptomatik

- Veränderungen der Selbstregulation bzw. Emotionskontrolle und Anpassungen der Regulation des Wohlbefindens

Neben den klassischen Formen der Psychotherapie sollte auch die Psychoedukation, insbesondere der Einbezug des sozialen Umfelds, berücksichtigt werden. Dies kann z.B. gegen schambehaftete Rückzugstendenzen helfen oder dem sozialen Umfeld ermöglichen, besser auf die spezifischen Bedürfnisse der Betroffenen einzugehen. Ähnliche Empfehlungen bestehen auch für die Depression im Alter [5].

Bisher fehlt für die Psychoanalyse im hohen Alter bei Personen mit psychotischer Störung die Evidenz. Allerdings kann bis ins hohe Alter durch die Plastizität unseres Gehirns das Beziehungserleben neu gestaltet und erfahren werden, weshalb der psychoanalytische Ansatz Raum und Zeit bis ins hohe Alter bieten kann, um den Alterungsprozess nicht als bedrohliche Wiederholung frühester Isolationserlebnisse zu erleben, sondern vielmehr im hohen Alter ggf. erstmals eine fördernde Umwelt zu erfahren. Das Annehmen von Hilfe während des psychoanalytischen Prozesses kann zu verbesserter Lebensqualität nicht nur für die Betroffenen, sondern auch für ihr pflegendes Umfeld führen. Das heutige Wissen um die Plastizität des Gehirns bis ins hohe Alter ist die wesentliche Grundlage für die Wirksamkeit der psychoanalytischen Psychotherapie über die Lebensspanne. Dank funktionaler Beziehungsgestaltung können ein verändertes Beziehungserleben, veränderte Denkmuster und Verhaltensweisen das Vertrauen und das emotionale Erleben verbessern und so zu verbesserter Lebensqualität im Alter führen.

\section{Psychosoziale Interventionen}

\section{Pflege von Menschen mit einer psychotischen Erkrankung im Alter}

Menschen mit einer schweren psychotischen Erkrankung erreichten in der Vergangenheit aufgrund ihrer ausgeprägten Multimorbidität das höhere Lebensalter nur selten. Im Kontext der demografischen Entwicklungen und der verbesserten Behandlungsangebote erhöht sich die Lebenserwartung von Menschen mit einer schweren psychotischen Erkrankung [11]. Zudem steigt der Anteil der Menschen, bei denen im höheren Alter z.B. aufgrund von Demenz, Delir etc. zum ersten Mal akut psychotische Symptome auftreten. Für ältere Menschen mit einer chronischen Störung aus dem schizophrenen Formenkreis haben sich pflegerische/psychosoziale Interventionen im klinischen Alltag bewährt [11]. Es gibt jedoch erst wenige systematische Behandlungsstudien für die meisten der basalen psychosozialen Therapieverfahren im Alter.

In der Auseinandersetzung mit einer evidenzbasierten Behandlung psychotisch erkrankter Menschen im Alter ist zu berücksichtigen, dass die Haltung der Verantwortlichen in den Institutionen, die Zusammensetzung und das $\mathrm{Zu}$ sammenspiel des Behandlungsteams die Grundlage psychosozialer Interventionen bilden. Sie wirken sich auf die Gestaltung der therapeutischen Beziehung aus und prägen sie [11]. Durch die Modellierung der strukturellen und organisatorischen Umgebungsbedingungen, wie z.B. die $\mathrm{Zu}$ sammensetzung des therapeutischen Teams, die fachliche Kompetenz der Mitarbeitenden, die Ausstattung der Abteilungs- und Therapieräume oder die Institutions- und Abteilungsregeln, kann dies auf Systemebene erreicht werden.

Bei der Behandlung älterer Menschen gilt es, die medizinischen Aspekte zu berücksichtigen, aber insbesondere aus pflegerischer Perspektive auch ein Verständnis und Raum für die Lebenserfahrung der Betroffenen zu schaffen [20]. Bei der Planung psychosozialer Interventionen sind deshalb die physischen Beeinträchtigungen, die veränderte Wahrnehmung von Zeit, Raum und Person sowie die im und mit dem Alter eintretenden somatischen Erkrankungen und veränderten Sinnbezüge und existenzielle Fragen zu integrieren [21, 22]. Psychosoziale Interventionen für ältere Menschen mit einer psychotischen Störung zielen darauf ab, die individuellen Möglichkeiten der Betroffenen zu erhalten, damit sie möglichst lange in ihrer sozialen Umgebung bleiben und am gesellschaftlichen Leben teilhaben können [11]. Dies wird durch die Modellierung der Umgebungsbedingungen erreicht, wie auch durch individuelle person-zentrierte Massnahmen, die auf die Stärkung verschiedener Lebensbereiche abzielen $[11,20,21,22,23,24]$. Als person-zentriert werden Ansätze bezeichnet, welche die Person und nicht die Erkrankung ins Zentrum stellen. Dabei werden sowohl die Individualität und die Autonomie als auch die fundamentale Eingebundenheit in zwischenmenschliche Beziehungen der Person hervorgehoben [22].

Die Bedürfnisse älterer Menschen müssen im Vordergrund stehen und die Planung der Interventionen soll auf 
dieser Basis geschehen. Einzel-, Gruppen- und milieutherapeutische Massnahmen sind altershomogen zu gestalten, und im Ablauf soll genügend Zeit mit Pausen eingeplant werden $[21,22,24,25]$. Der Aufbau einer verlässlichen und wertschätzenden Beziehung, die Sicherheit vermittelt und auf Vertrauen basiert, ist notwendig bei der Pflege von älteren Menschen mit einer psychotischen Erkrankung. Diese benötigt Zeit, Gespräche und Raum für «nicht-funktionale» Begegnungen [25, 26], die nicht während einer Pflegeverrichtung geschehen, sondern in denen es um eine zwischenmenschliche Interaktion geht $[21,26]$, in Form von Gesprächen, die offen sind für das, was geschieht und die Betroffenen im Alltag und während der Behandlung bewegt. Dabei sollten die für die Person bedeutsamen biografischen und wesentlichen Alltagsthemen einbezogen werden. Das Erleben von Alter,
Krankheit und Krisen wirft Fragen nach dem Sinn des Lebens auf. Im Alter verändern sich Ziele und Sinnfragen, die Akzeptanz des eigenen Lebens wird bedeutender. Dabei sollen Themen wie Lebenssinn, Lebensüberdruss, Glauben und Spiritualität offen angesprochen werden und ihren Raum erhalten.

Der Miteinbezug in Tages- und Gruppenaktivitäten auf den Abteilungen und/oder aktive Teilnahme an sozialen Aktivitäten ist nach Belastbarkeit der Betroffenen zu gewährleisten [24].

Die motivierende Gesprächsführung (MI) ist bei all den Interventionen eine wichtige kommunikative Technik zur Stärkung der Beziehung mit den Betroffenen. Sie geht davon aus, dass z.B. ablehnende Verhaltensweisen nicht auf einer fehlenden Motivation oder auf Widerstand beruhen, sondern dass es dafür Gründe gibt, die ermittelt und be-

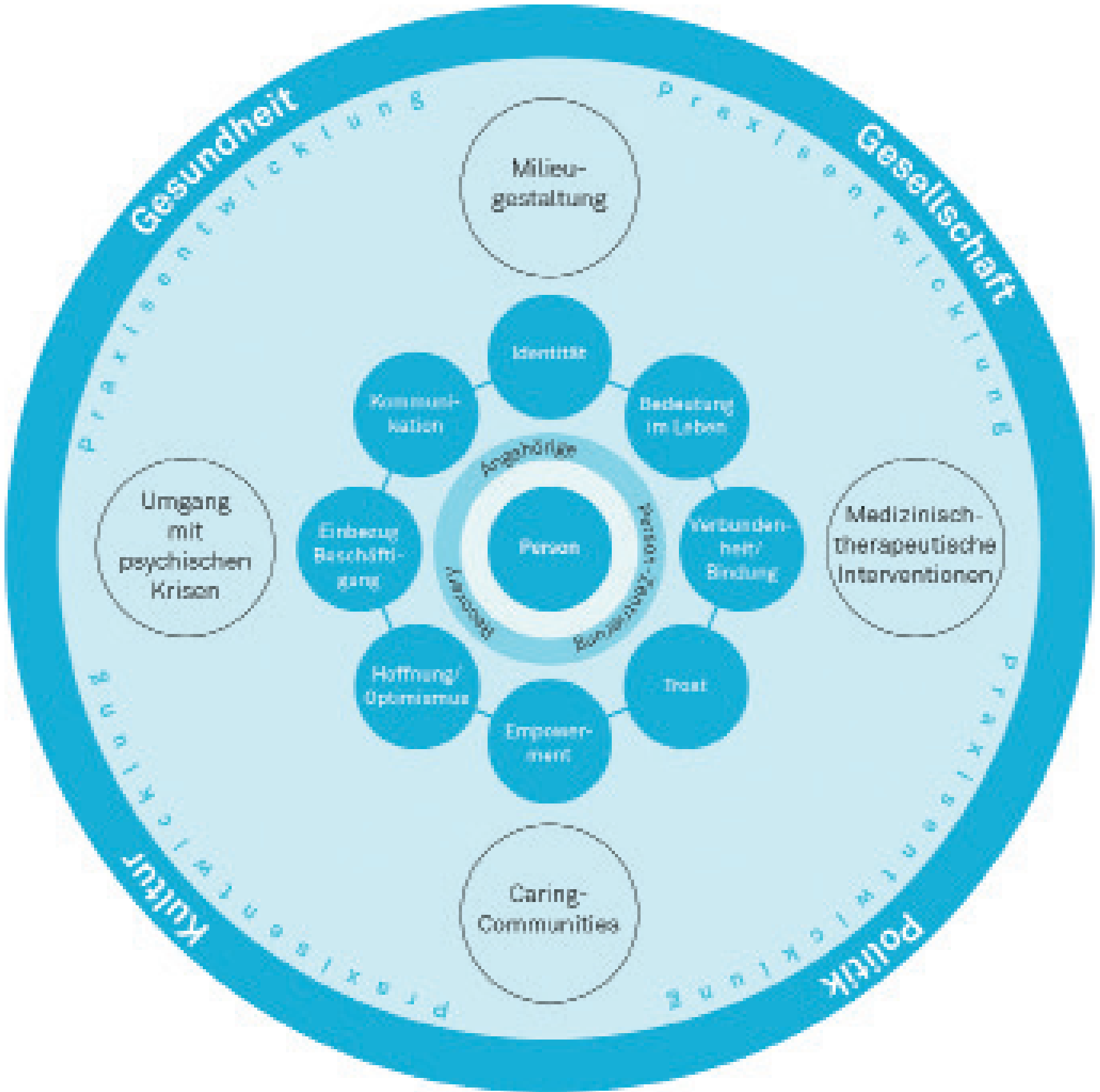

Abbildung 1. Psychosoziale Interventionen, eigene Darstellung: Lötscher, Hirsbrunner, Vögeli, Neyenhuys \& Roth, 2021. 
rücksichtigt werden müssen in der Behandlung. Pflegefachpersonen begleiten die Betroffenen partnerschaftlich, um sie in ihrer Selbstwirksamkeit zu fördern und Entscheidungen gemeinsam $\mathrm{zu}$ antizipieren und zu tragen [27]. Eine bildliche Zusammenfassung bietet die Abbildung 1. Die Vernetzung und Zusammenhänge der erwähnten Schwerpunkte sowie deren Komplexität soll damit verdeutlicht werden.

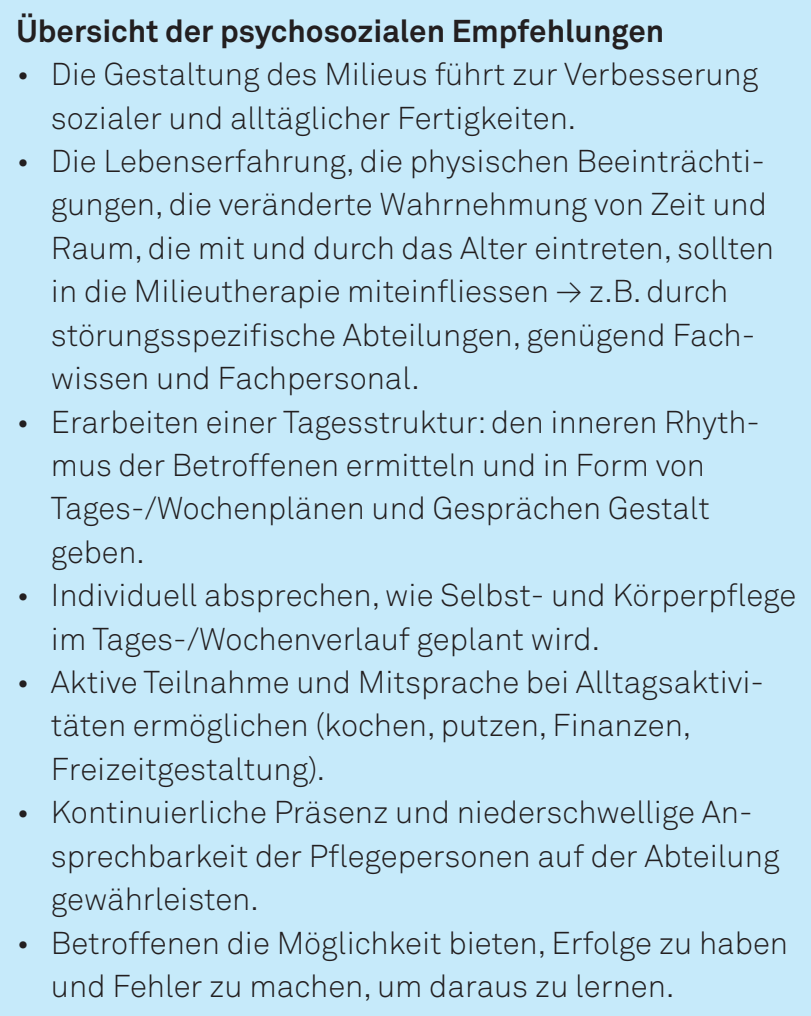

\section{Pflegetherapeutische Interventionen}

Die Wahl des Behandlungskonzepts richtet sich nach klinischen Faktoren wie der Symptomschwere, dem Erkrankungsverlauf unter Berücksichtigung der individuellen Komorbiditäten oder Folgeerkrankungen sowie der Patientinnenpräferenz. Basierend auf den S3-Leitlinien zur Psychosozialen Therapie [11] sollten spezifische pflegeund medizintherapeutische Interventionen im Alter folgende Bereiche umfassen:

- Psychoedukative Interventionen unter Einbeziehung der Angehörigen

- Medizintherapeutische Therapien inklusive Ergotherapie, Aktivierungstherapie, Kunsttherapie, Bewegungstherapie etc.

- Milieutherapie, Inklusionsförderung, Training sozialer und kognitiver Fertigkeiten und Alltagskompetenzen in Form von Einzel- und Gruppenangeboten

- Leitlinienorientierte manualisierte psychotherapeutische Interventionen wie Integriertes Psychologisches Therapieprogramm oder Kognitive Verhaltenstherapie
Ein wesentlicher Beitrag zu den psychotherapeutischen Therapien durch die Pflegefachpersonen ist das Trainieren und Adaptieren der therapeutisch erarbeiteten Massnahmen mit den Betroffenen ihn ihren jeweiligen Alltag.

\section{Psychosoziale Interventionen im Familiensystem}

Die Krankheit einer Person beeinflusst nicht nur das Individuum, sondern das ganze soziale System. Das Krankheitsgeschehen einer Person kann somit in den Mittelpunkt des Lebens der ganzen Familie rücken und das ganze System verändern. Gleichzeitig haben die Angehörigen einen wesentlichen Einfluss auf den Gesundheitszustand und das Wohlbefinden jedes einzelnen Familienmitglieds [28, 29, 30, 31]. Die Unterstützung von Familien und Angehörigen, die Zusammenarbeit sowie der Miteinbezug Familienangehöriger ist ein wesentlicher Bestandteil psychosozialer Betreuung [11].

Familien und Angehörige tragen massgebend zur Stabilität von psychisch erkrankten Menschen bei [11]. Sie bieten langjährige Unterstützung und Begleitung in der Therapie, der Gestaltung des Alltags und im Umgang mit der psychischen Erkrankung. Diese Rolle, die Erwartungen und Zuschreibungen sowie Anforderungen, können für Angehörige mit Belastungen auf verschiedenen Ebenen einhergehen [11, 32, 33]. Angehörige sind somit aufgrund ihrer besonderen Position im Familiensystem auch Mitleidende.

Die Familie als System wahrzunehmen bedeutet, dass die Familienmitglieder von Anfang an als Mitbetroffene in die Behandlung einbezogen werden. Um gezielt systemisch, familienzentriert betreuen zu können, sollten die Bedürfnisse, Ressourcen und Belastungen von Kranken und Angehörigen hinsichtlich der pflegerischen Versorgung miterhoben werden $[5,11,29,34,35,36]$.

Psychoedukative Aspekte und angehörigengestützte Verfahren sollten eingesetzt werden, um damit das nötige Krankheitsverständnis zu entwickeln oder zu aktualisieren und Belastungen zu reduzieren. Dies kann nachweislich zur Verbesserung der Interaktionen mit den Betroffenen beitragen und Konflikte vermeiden oder abbauen. Auch haben sich stabilisierende Gespräche mit Angehörigen bei psychischen oder physischen Veränderungen als erfolgsversprechend erwiesen $[5,11,29,36,37]$.

Die Teilnahme an Angehörigengruppen oder individuellen Unterstützungsangeboten, allenfalls Selbsthilfeangebote oder Trialoge sollten empfohlen werden [11]. Hierfür sind die Stiftung Pro Mente Sana zu nennen, die sich für psychisch beeinträchtige Menschen und deren Angehörige in der Schweiz einsetzt, sowie der Verein Netzwerk Angehörigenarbeit Psychiatrie (NAP). Beide Vereinigungen haben Angebote wie beispielsweise Gruppen für Angehörige. Bekannte und als wirkungsvoll geltende Angehörigengruppen sind jene zu den behavioralen und psychologischen Symptomen der Demenz sowie zu Depression im Alter [5, 29, 30, 35].

Die Empfehlungen zu den psychosozialen Interventionen sind in Abbildung 1 dargestellt und im Textkasten 1 zusammengefasst. 


\section{Pharmakotherapie}

Medikamentöse Therapien bei der Psychose sollten im Alter wegen der oft schlechteren Verträglichkeit und der oft bereits vorbestehenden Polypharmazie zurückhaltend eingesetzt werden.

In der Praxis kann die Auswahl geeigneter Psychopharmaka entsprechend dem in Abbildung 2 dargestellten Algorithmus erfolgen.

Wenngleich Antipsychotika die wichtigste Medikamentengruppe darstellen, kommen auch Benzodiazepine bei psychotischen Störungen zum Einsatz. Die wichtigsten Indikatoren sind in Tabelle 4 dargestellt und entsprechen im Wesentlichen den Empfehlungen für jüngere Menschen [3]. Die Evidenzlage zur Ableitung spezifischerer Empfehlungen für Ältere ist praktisch völlig fehlend. Besonders zu beachten sind jedoch die negativen Auswirkungen auf die kognitive Leistung und u.a. auf die Muskelkraft, was zu einer erhöhten Sturzgefahr führt.

\section{Interventionelle Verfahren}

Derzeit existieren verschiedene interventionelle Verfahren. Von grösster Bedeutung ist dabei die Elektrokonvulsionstherapie (EKT), die ursprünglich bei Psychosen eingesetzt wurde, und wofür es sehr gute Evidenz gibt. Dazu gibt es auch die beste Evidenz. Im Hinblick auf die Behandlung von Psychosen bei Älteren lassen sich die folgenden Indikationen abgrenzen:

- Behandlung von (therapieresistenter) Depression mit psychotischen Symptomen

- Behandlung von therapieresistenter, u.a. Clozapinresistenter, Schizophrenie

- Behandlung von Katatonie (inkl. perniziöse Katatonie, malignem Antipsychotika-Syndrom)

- Behandlung von schwerer Suizidalität

- Augmentation der Psychopharmakotherapie, sofern die somatischen Komorbiditäten das erfordern (z.B. kein Lithium bei eingeschränkter Nierenfunktion)

- Seltener zur Behandlung von starker Erregung

Kriterium 1: Wirkspektrum (welches Wirkspektrum benötige ich für die Behandlung: sedierend/nicht sedierend, hohe antipsychotische Potenz bzW. Wirkung auf Negativsymptomatik?)

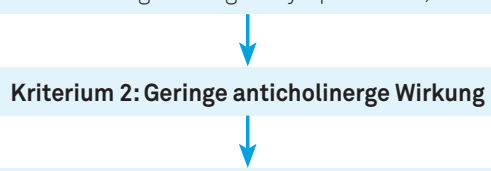

Kriterium 3: Medikamenten Auswahl bei Risikofaktoren/Komorbiditäten:

- QTc-Intervall-Verlängerung: keine oder geringe QTc-Intervall-Verlängerung bei Lurasidon, Brexpiprazol, Aripiprazol, Paliperidon, Haloperidol - Zerebrovaskuläre Ereignisse/Risikofaktoren in der Vorgeschichte: Cave: Haloperidol, Olanzapin, Risperidon; Günstiger: Aripiprazol, Quetiapin - Gewichtszunahme, Stoffwechselstörungen, metabolisches Syndrom: Eher gering bei Lurasidon, Haloperidol, Aripiprazol, Brexpiprazol, Cariprazin - Leber-/Nierenfunktionsstörungen: Ausscheidung vorwiegend renal: Amisulprid, Paliperidon

Falls eine Monotherapie wegen des Nebenwirkungsprofils nicht möglich ist, sind ggf. zwei Substanzen mit sich ergänzendem Wirkprofil und in der Summe günstigerem Nebenwirkungsprofil als unter Monotherapie zu wählen.

Abbildung 2. Algorithmus: Antipsychotikaauswahl bei Psychosen im Alter.

Tabelle 4. Indikationen für Benzodiazepine bei psychotischen Symptomen

\begin{tabular}{ll}
\hline Symptomatik & Empfehlungen \\
\hline Allgemeine pharmakotherapeutische Empfehlungen & \\
\hline Akute Phase & $\begin{array}{l}\text { Erstlinien-Reservebehandlung zur Beruhigung bei Angstsymptomen in den } \\
\text { ersten Wochen der stationären Behandlung (maximal 3-4 Wochen) }\end{array}$
\end{tabular}

Pharmakotherapeutische Empfehlungen für spezifische Situationen

Katatone Symptomatik

In Fällen von Heteroaggressivität
Pharmakologische Behandlung erster Wahl

Zur medikamentösen Behandlung, die auf eine schnelle Beruhigung abzielt, hat sich die orale Verabreichung von Risperidon, Olanzapin oder Haloperidol als wirksam erwiesen. Diese Medikamente können mit Benzodiazepinen kombiniert werden.

Bei Akathisie haben sich Benzodiazepine als wirksam erwiesen.

Die Verabreichung von Benzodiazepinen kann für eine kurze Dauer (wenn möglich nicht länger als 3-4 Wochen) gerechtfertigt sein zuständen, die nicht einfach auf eine primäre Psychose reduziert werden können und eine spezifische Behandlung erfordern 
Die Wirksamkeit von EKT steigt mit dem Alter eher an. Relevant sind jedoch die typischerweise transienten kognitiven Defizite, die bei unilateraler Stimulation geringer als bei bilateraler EKT ausfallen. Eingeschränkte Kognition ist keine Kontraindikation gegen EKT. Tatsächlich können vor EKT bestandene kognitive Einbussen nach erfolgreicher Stimulationsserie deutlich zurückgehen, sofern diese aufgrund der depressiven bzw. psychotischen Störung bestanden [38].

In den vergangenen Jahren hat die repetitive transkranielle magnetische Stimulation (rTMS) an Bedeutung gewonnen. Die bisherigen Studien konzentrieren sich aber auf den Einsatz bei nicht-geriatrischen Personen mit Depression, sodass insbesondere zur Wirksamkeit bei älteren Menschen bisher die Evidenz fehlt.

\section{Zusammenfassung}

- Personenzentrierung und Recovery prägen die multiprofessionelle Behandlung von Menschen mit einer psychotischen Erkrankung im Alter.

- Psychotische Störungen sind im Alter häufig und treten vor allem bei Depressionen, Demenzen, Substanz- und Medikamentenmissbrauch und Delirien auf.

- Neben der psychiatrischen Untersuchung und dem Somato-/Neurostatus spielen die Erfassung des Funktionsniveaus im alltäglichen Leben sowie die Erhebung der Fremdanamnese eine wesentliche Rolle.

- Kranke sind immer im Kontext ihres Lebensumfelds mit ihren Angehörigen zu verstehen.

- Therapieziele müssen in Absprache mit dem Patienten/ der Patientin festgelegt werden.

- Bei älteren Menschen liegt der Fokus der Behandlung auf dem Erhalt der Autonomie, der Selbstständigkeit, dem Wohlbefinden und der subjektiven Lebensqualität.

- Psychotherapeutische Interventionen sind auch im Alter wirksam, sollten jedoch individuell an die Person adaptiert werden.

- Bei pharmakologischen Interventionen steht die Verträglichkeit im Vordergrund. Zu vielen Substanzen fehlen Studien bei älteren Menschen.

- Bei den interventionellen Verfahren steht die EKT mit guter Wirksamkeit und in der Regel auch Verträglichkeit im Vordergrund.

\section{Bibliografie}

1. Klöppel S, Savaskan E. Psychosen im Alter. 1. Auflage. Bern; Hogrefe: 2021.

2. DGPPN. S3-Leitlinie Schizophrenie - Konsultationsfassung. 2018.

3. Kaiser S, Berger G, Conus P, et al. Die SGPP-Behandlungsempfehlungen zur Schizophrenie. Swiss Medical Forum. 20. Juni 2018;18(25):532-539.
4. Savaskan E, Baumgartner M, Georgescu D, et al. Empfehlungen zur Prävention, Diagnostik und Therapie des Delirs im Alter. Praxis (Bern 1994). 2016;105(16):941-952.

5. Hatzinger M, Hemmeter U, Hirsbrunner T, et al. Empfehlungen für Diagnostik und Therapie der Depression im Alter. Praxis. 2018;107(3):127-144.

6. Savaskan E, Bopp-Kistler I, Buerge M, et al. [Recommendations for diagnosis and therapy of behavioral and psychological symptoms in dementia (BPSD)]. Praxis (Bern 1994). 2014;103(3):135-148.

7. Reinhardt MM, Cohen Cl. Late-life psychosis: diagnosis and treatment. Curr Psychiatry Rep. 2015;17(2):1.

8. Brodaty H, Luscombe G, Parker G, et al. Increased rate of psychosis and psychomotor change in depression with age. Psychol Med. 1997;27(5):1205-1213.

9. Bürge M, Bieri G, Brühlmeier M, et al. Recommendations of Swiss Memory Clinics for the Diagnosis of Dementia. Praxis (Bern 1994). 2018;107(8):435-451.

10. Savaskan E, Hasemann W. Leitlinie Delir: Empfehlungen zur Prävention, Diagnostik und Therapie des Delirs im Alter [Internet]. Bern; Hogrefe Verlag: 2017. https://www.zora.uzh.ch/id/ eprint/147208; letzter Zugriff: 23.01.2020.

11. DGPPN. S3-Leitlinie Psychosoziale Therapien bei schweren psychischen Erkrankungen. 2. Auflage. Berlin; Springer: 2019.

12. James IA, Jackman L. Herausforderndes Verhalten bei Menschen mit Demenz. 2. Auflage. Bern; Hogrefe: 2019.

13. Klöppel S, Savaskan E, Kuhn R, et al. [Recognition of Psychiatric Symptoms in Inpatient Long-Term Care]. Praxis (Bern 1994). 2020;109(4):301-308.

14. Schwarze T, Steinauer R, Beeri S. Somatische Pflege in der psychiatrischen Arbeit. 1. Auflage 2019. Köln: Psychiatrie: 2019.

15. Richter D, Gühne U, Stein J, Weinmann S, Becker T, RiedelHeller SG. Die Effekte nicht-pharmakologischer, gesundheitsfördernder Interventionen bei Menschen mit schweren psychischen Erkrankungen. Psychiat Prax. 2018;45(07):347-357.

16. Marengoni A, Rizzuto D, Wang H-X, Winblad B, Fratiglioni L. Patterns of chronic multimorbidity in the elderly population. J Am Geriatr Soc. 2009;57(2):225-230.

17. Granholm E, McQuaid JR, McClure FS, et al. A randomized, controlled trial of cognitive behavioral social skills training for middle-aged and older outpatients with chronic schizophrenia. Am J Psychiatry. 2005;162(3):520-529.

18. Berry K, Barrowclough C. The needs of older adults with schizophrenia: implications for psychological interventions. Clin Psychol Rev. 2009;29(1):68-76.

19. Maercker A. Alterspsychotherapie und klinische Gerontopsychologie. Berlin; Springer: 2015.

20. World Health Organization. Promoting recovery in mental health and related services: WHO quality rights training to act, unite and empower for mental health. [Internet]. 2017. https://www. who.int/mental_health/policy/quality_rights/QRs_flyer_ eng_2017.pdf?ua=1; letzter Zugriff: 18.08.2021.

21. DNQP. Expertenstandard Beziehungsgestaltung in der Pflege von Menschen mit Demenz [Internet]. 2018. https://www. dnqp.de/fileadmin/HSOS/Homepages/DNQP/Dateien/Experten standards/Demenz/Demenz_AV_Auszug.pdf; letzter Zugriff: 18.08.2021.

22. Kitwood T. Demenz. Der person-zentrierte Ansatz im Umgang mit verwirrten Menschen. Bern; Hogrefe: 2019.

23. Naldemirci Ö, Lydahl D, Britten N, Elam M, Moore L, Wolf A. Tenacious assumptions of person-centred care? Exploring tensions and variations in practice: Health [Internet]. 2016. https://journals.sagepub.com/doi/10.1177/13634593166 77627; letzter Zugriff: 10.01.2020.

24. Voogt LA. Providing structure: unraveling and building a psychiatric nursing intervention. [Nederland]: [Uitgever niet vastgesteld]; 2016.

25. Richter D, Schwarze T, Hahn S. Merkmale guter psychiatrischer Pflege und Betreuung. Psych Pflege. 2010;16(01):17-20.

26. Slade M. Everyday solutions for everyday problems: how mental health systems can support recovery. Psychiatr Serv. 2012;63(7):702-704. 
27. Miller WR, Rollnick S. Motivierende Gesprächsführung [Internet]. 2015. https://www.lambertus.de/assets/adb/b8/b85cbab ff1d9965b.pdf; letzter Zugriff: 18.08.2021.

28. Friedemann M-L. Familien und umweltbezogene Pflege. Die Theorie des systemischen Gleichgewichts. Bern; Hogrefe: 1996.

29. DEGAM Pflegende Angehörige von Erwachsenen [Internet]. 2019. https://www.degam.de/files/Inhalte/Leitlinien-Inhalte/ Dokumente/DEGAM-S3-Leitlinien/053-006_Pflegende\%20 Angehoerige/053-006 L_DEGAM\%20LL\%20Pflegende\%20 Angeho\%CC\%88rige_4-3-2019.pdf; letzter Zugriff: 18.08.2021.

30. Schoppmann S, Schnepp W. Bewältigungsstrategien von Angehörigen depressiv erkrankter Menschen [Internet]. 2016. https://www.rosenfluh.ch/psychiatrie-neurologie-2016-04/ bewaeltigungsstrategien-von-angehoerigen-depressiverkrankter-menschen; letzter Zugriff: 18.08.2021.

31. Weddinger Modell. Köln; Psychiatrie Verlag: 2014

32. Heumann K, Janßen L, Ruppelt F, Mahlke C, Sielaff G, Bock T. Auswirkungen von Peer-Begleitung für Angehörige auf Belastung und Lebensqualität. Z Psychiatr Psychol Psychother. 2016;64(1):45-53.

33. Unterstützung für betreuende und pflegende Angehörige Situationsanalyse und Handlungsbedarf für die Schweiz Bericht des Bundesrats [Internet]. 2014. http://caregiver-center.ch/ wp-content/uploads/2019/08/bericht_des_br_zur_angehoerig enpflege_de.pdf; letzter Zugriff: 18.08.2021.

34. Akgun-Citak E, Attepe-Ozden S, Vaskelyte A, et al. Challenges and needs of informal caregivers in elderly care: Qualitative research in four European countries, the TRACE project. Arch Gerontol Geriatr. 2020;87:103971.

35. Schizophrenia Carer Playbook European Federation of Associations of Families of People (EUFAMI) [Internet]. http://eufami. org/wp-content/uploads/2019/02/schizophrenia_carer_ playbook.pdf; letzter Zugriff: 18.08.2021.
36. Manike K, Kraft E. Bedürfnisse von Angehörigen mit psychisch erkrankten suizidalen Personen in ihrem Umfeld: Empfehlungen zur Unterstützung der Angehörigen für Kliniken und Fachpersonen im Bereich der stationären Psychiatrie [Internet]. 2020. Verfügbar unter: https://www.bag.admin.ch/bag/de/ home/strategie-und-politik/politische-auftraege-und-aktions plaene/aktionsplan-suizidpraevention/suizidpraeventionpsychiatrische-versorgung.html; letzter Zugriff: 18.08.2021.

37. Sanghani SN, Petrides G, Kellner CH. Electroconvulsive therapy (ECT) in schizophrenia: a review of recent literature. Curr Opin Psychiatry. 2018;31(3):213-22.

\section{Historie}

Manuskript eingereicht: 06.07.2021

Manuskript akzeptiert: 15.07.2021

\section{Dank}

Die Autorinnen und Autoren danken Frau Sandra Habegger, UPD Bern, für die Unterstützung bei dem Verfassen dieses Manuskripts und der Buchversion.

\section{Interessenskonflikte}

Es bestehen keine Interessenskonflikte.

\section{Prof. Dr. med. Stefan Klöppel, M.D.}

Direktor und Chefarzt

Universitäre Psychiatrische Dienste Bern

Murtenstrasse 21

3008 Bern

stefan.kloeppel@upd.unibe.ch 